\title{
Expressing (un)certainty in no uncertain terms: Reply to Fox and Ülkümen
}

\author{
Karl Halvor Teigen ${ }^{1,2}$ and Erik Løhre ${ }^{1,2}$ \\ ${ }^{1}$ Simula Research Laboratory, Oslo \\ ${ }^{2}$ Department of Psychology, University of Oslo
}

The ways people estimate and express degrees of uncertainty and probability have fascinated philosophers, statisticians, cognitive psychologists, and domain experts (for instance in medicine, military intelligence, and climate science) for years. What makes people think that one outcome, or one assertion, is more likely to be true than another, and how well do people's estimates correspond to normative criteria? Further questions, more relevant to the present discussion, are: How are their estimates expressed in words, instead of or in addition to numbers, and how are these expressions understood (or misunderstood) by recipients? Until recently, most work within this field has revolved around the degree of certainty or probability implied by selected phrases and terms. A recurrent conclusion of this research has been that verbal phrases are vague, in the sense that the same term (e.g., a chance, not certain) can be used to characterize a whole range of numerical probabilities (Budescu \& Wallsten, 1995).

But natural language is a supple medium, which despite its alleged vagueness can convey a host of semantic and pragmatic implications, including hedges (maybe) and outcome valence (a hope vs. a risk). One aspect of verbal probability expressions that has been identified and studied extensively is their directionality (Honda \& Yamagishi, 2006; Teigen \& Brun, 1995, 1999). Whereas some verbal phrases direct our attention towards an explicit target event (it is quite possible Donald Trump will build a wall on the Mexican border), others point to the target event's non-occurrence (it is uncertain the wall will be built). Authors discussing the whole gamut of verbal expressions waver accordingly between a positive label ("verbal probabilities") or a negative one ("variants of uncertainty”), and sometimes end up with a combination of both, as do Ülkümen, Fox, and Malle (2016) when they use the negative term uncertainty as a generic name to include two positive terms, confidence and likelihood. Choice of labels is not easy. 
The present discussion revolves around another, equally fascinating aspect, namely in which ways, how well, or how vaguely, the nature and origin of these likelihoods or uncertainties are reflected in language. Do we think of them as beliefs located within our heads, or as propensities and regularities in the environment? Can they be attributed to our internal (imperfect) state of knowledge, or rather to characteristics (trends, frequencies, and variabilities) of the external world? Philosophers of science will recognize this as reflecting epistemic vs. aleatory probability concepts that have been around for a very long time (Hacking, 1975). However, empirical studies of how these concepts are reflected in lay people's thinking have been scarce, as have studies of how they are reflected in language until now, when it appears that our work and that of Fox and Ülkümen and their colleagues have developed more or less in parallel. We think that these new streams have the potential to open up a rich field of research, accompanied by some divergences and disputes that rise unavoidably from doing independent science.

Note for instance the divergence in the choice of labels: although both parties start from the same underlying probability concepts, we use the terms internal vs. external uncertainty, while Fox and Ülkümen have chosen the labels epistemic vs. aleatory uncertainty. This may reveal a difference in the view on what the key distinction is between these concepts, and may also explain the background of the current discussion.

Epistemic uncertainty, according to Fox and Ülkümen, can also be called "knowable" uncertainty, and is attributed to missing knowledge, skill or information. Aleatory uncertainty, on the other hand, has the alternative label "random" uncertainty, and stems from chance or stochastic processes. Fox and Ülkümen assume that this distinction is reflected in natural language by different words. Together with collaborators, they have performed several intriguing studies that were unknown to us at the time we first performed and submitted our study. Their studies convincingly show that some terms (or stems) are primarily used to describe mental states (e.g., "confidence") whereas others are better suited to describe external phenomena characterized by variability and randomness (e.g., "chance”).

Our analysis led us to believe that the distinction between the two types of uncertainty is more directly mirrored at the clause level, specifically by the grammatical and/or logical subject of the statement. Who, or what, is certain or uncertain? Is it a 
sentient being, describing his or her beliefs, or are we speaking about states of the external world? We talk in our paper about internally focused vs. externally focused statements, but it could also be referred to as a distinction between personal vs. impersonal uncertainty, or subjective vs. objective, as Fox and Ülkümen do in their comment. However, this is not a first vs. third person distinction, because "I am sure" or "she is sure" both refer to the mental states or beliefs of sentient beings. Consider the statement: "It is almost certain/very probable that Petter Northug will win at least one gold medal in the Olympics". Here "it" is an impersonal pronoun, which in this context can be regarded as an anticipatory subject, because the logical subject is to be found in the embedded clause (Kaltenböck, 2003). "It” refers to Petter Northug's victory (an external event), which is described as almost certain or as very probable, as the case may be.

So there is a slight difference in emphasis, and a difference in assumptions about which aspect of language (clauses or terms) offers the most promising lead for reflecting different attributions of uncertainty. From our divergent approaches a new question emerges, highlighted by Fox and Ülkümen's present commentary. How are these two aspects of language related? Can any subject go along with any term? This would, of course, be ideal for an experimental psychologist who would like to treat them as independent factors in a factorial design, like the one that Fox and Ülkümen would have liked us to produce, and (since we did not do so) have provided themselves in their Comment.

Unfortunately, language does not consist of elements that can be combined and recombined arbitrarily according to a 2 x 2 design. Some terms used to describe uncertainty require personal subjects, whereas others require impersonal ones. Take verbs. Some (un)certainty verbs are epistemic, like I know, I believe, I think, I guess, I doubt. The subject here has to be a sentient being, and these verbs can accordingly only be preceded by a personal and not by an impersonal pronoun. When speaking of external events, we use different verbs, like it will happen, it can occur, it may be the case. In such statements it does not make sense to exchange "it" with a personal pronoun. When it comes to adjectives, adverbs, and nouns, there are some that naturally describe mental states, and are easily combined with a first person pronoun as a subject. "I am confident 
that...” requires somebody to be confident. Others refer to states of the world. The anticipatory (dummy) subject "it” or "there” in “There is a small chance / a possibility / probability that..." cannot be replaced by a personal pronoun. We think that the terms certain and uncertain are special in their ability to accommodate both personal and impersonal subjects, but by doing so, the meaning of (un)certainty is transformed, and changes its referent from describing an internal to an external state. It may well be that certain is predominantly used to characterise epistemic states, as suggested by Fox and Ülkümen's recent work. However, it can also describe environmental facts and is then used (almost) interchangeably with probability, as we show in our Experiment 4. (Further studies may, however, unravel a difference since improbable and uncertain are not the same.)

In their attempt to vary term and subject orthogonally, Fox and Ülkümen make use of two stems they claim to be of the confidence (epistemic) kind and two that they claim are of the likelihood (aleatory) type. These stems are then crossed with subjective ( $\mathrm{I} \mathrm{am}$ ) and objective (it/there is) language. We see two problems with this design. One is that certain is not a pure descriptor of epistemicness, but changes its meaning according to its logical subject, as discussed above. The other resides in the (in our view not very successful) attempt to pair a personal subject with an aleatory term. Since the phrase "I am an $80 \%$ probability that...” is linguistically unacceptable, subjective language has been preserved by adding a subjective speaker: "I think there is an $80 \%$ probability that...”. But this statement contains two clauses, not one. The first is subjective about a speaker, who thinks something, whereas the second follows a recognizable objective template starting with "there is". We claim accordingly that the study does not fully succeed in crossing subject with term.

In an earlier version of their commentary, the authors reported a study where the statements' locations on the aleatory/epistemic dimension were measured by their scores on the EARS (a 10-item scale previously used by Ülkümen, Fox, \& Malle, 2016). In the study reported in the present commentary, this scale is replaced by a single item focusing on whether the speaker is using singular or distributional reasoning. We find the distinction between singular and distributional more appropriate (as discussed by Kahneman and Tversky, 1982) and also easier to conceptualize as independent of the 
subjective/objective distinction. We are also pleased to see that the results displayed in Tables 2-3 indicate that our distinction between first person and impersonal pronouns led to strong effects on the subjective/objective dimension.

Some conceptual and terminological disagreement remains. We still feel it odd to describe all singular probabilities as "epistemic". What is left out of this taxonomy is singular probabilities that are based on causal considerations of objective features in the external world, what Popper (1959) and Kahneman and Tversky (1982) have described as a propensity interpretation of probabilities. The fact that the home team is stronger and thus will probably win the football match is not necessarily an "epistemic" question of degrees of knowledge, but can be attributed to an observable list of assets and strengths. This kind of singular probability can clearly be seen as external, although the label “aleatory”, which suggests randomness, may be a misleading one. In our view, epistemic

uncertainty is usually (if not exclusively) internal/subjective, while aleatory uncertainty is usually external/objective (note that Gillies (2000), in his book on philosophical theories of probability, makes a similar distinction between epistemic and objective probability concepts).

To sum up, we agree with Fox and Ülkümen that probabilistic communication depends both on type of clause and type of term, but peculiarities of language create difficulties for a successful crossed design. It seems to us that the term pair certain/uncertain is an especially interesting case that can be used in both an epistemic and an aleatory sense, and would welcome further studies focusing specifically on this two-faced term.

\section{References}

Budescu, D. V., \& Wallsten, T. S. (1995). Processing linguistic probabilities: General principles and empirical evidence. The Psychology of Learning and Motivation, 32, 275-318. doi:10.1016/S0079-7421(08)60313-8

Fox, C. R. \& Ülkümen, G. (in press). Comment on Løhre and Teigen (in press). Thinking \& Reasoning.

Gillies, D. (2000). Philosophical theories of probability. London: Routledge. 
Hacking, I. (1975). The emergence of probability: A philosophical study of early ideas about probability, introduction and statistical inference. Cambridge, UK: Cambridge University Press.

Honda, H., \& Yamagishi, K. (2006). Directional verbal probabilities: Inconsistencies between preferential judgments and numerical meanings. Experimental Psychology, 53, 161-170. doi:10.1027/1618-3169.53.3.161

Kaltenböck, G. (2003). On the syntactic and semantic status of anticipatory it. English Language and Linguistics, 7, 235-255. doi:10.1017/S1360674303001096

Kahneman, D., \& Tversky, A. (1982). Variants of uncertainty. Cognition, 11, 143-57.

Løhre, E. \& Teigen, K. H. (2016). There is a 60\% probability, but I am 70\% certain: Communicative consequences of external and internal expressions of uncertainty. Thinking \& Reasoning, 22, 369-396. doi:10.1080/13546783.2015.1069758

Popper, K. R. (1959). The propensity interpretation of probability. British Journal for the Philosophy of Science, 10, 25-42

Teigen, K. H. \& Brun, W. (1995). Yes, but it is uncertain: Direction and communicative intention of verbal probabilistic terms. Acta Psychologica, 88, 233-258. doi:10.1016/0001-6918(93)E0071-9

Teigen, K. H. \& Brun, W. (1999). The directionality of verbal probability expressions: Effects on decisions, predictions, and probabilistic reasoning. Organizational Behavior and Human Decision Processes, 80, 155-190. doi:10.1006/obhd.1999.2857

Ülkümen, G., Fox, C. R., \& Malle, B. F. (2016). Two dimensions of subjective uncertainty: Clues from natural language. Journal of Experimental Psychology: General, 145, 1280 
